(C)2009 IEEE. Personal use of this material is permitted. However, permission to reprint/republish this material for advertising or promotional purposes or for creating new collective works for resale or redistribution to servers or lists, or to reuse any copyrighted component of this work in other works must be obtained from the IEEE. 


\title{
Medical Health Knowledge Management using Wireless Network: Moroccan Hospitals Case Study
}

\author{
Jamaly, S. ${ }^{1}$, Wongthongtham, P. ${ }^{2}$ and Aseeri, A. ${ }^{2}$ \\ ${ }^{1}$ Management School, Curtin University of Technology, Perth, Australia, e-mail: sanaa.jamaly@postgrad.curtin.edu.au \\ ${ }^{2}$ DEBI Institute, Curtin University of Technology, Perth, Australia, e-mails: P.Wongthongtham@cbs.curtin.edu.au, \\ a.aseeri@postgrad.curtin.edu.au
}

\begin{abstract}
Hospitals and medical health services are large business that we have to make them successful for their earned profits and incomes. Unifying the communication in hospitals can approve the environments and avoid the problems faced. Wireless networks are very useful in large crowded places like hospitals. Wireless technology has empowered the medical health systems by reducing the paperwork that nurses or doctors have to deal with them whole day. Its primary purpose is to ensure effective care of patients by increasing the accuracy and speed of healthcare. This paper highlights patients' knowledge management in hospitals using the wireless communication since the rate of medical errors has increased lately. The intention of this paper is to utilize an advanced technology to help hospitals raise the rate of care of patients throughout the use of mobile data collection device that transmits data to a centralized database to raise the visibility of data to the receivers.
\end{abstract}

Index Terms - knowledge management, $\mathrm{Wi}-\mathrm{Fi}$, wireless LAN, alerts management

\section{INTRODUCTION}

Wireless networks [1] are very useful in large crowded places like hospitals that need this technology applied into their systems. Wireless technology has empowered the medical health systems by reducing the paperwork that nurses or doctors have to deal with them whole day. Its primary purpose is to ensure effective care of patients by increasing the accuracy and speed of healthcare.

This paper highlights patients' knowledge management in hospitals using the wireless communication since the rate of medical errors has increased lately. The intention of this paper is to utilize an advanced technology to help hospitals raise the rate of care of patients throughout the use of mobile data collection device that transmits data to a centralized database to raise the visibility of data to the receivers.

This paper is organized as follows. First we discuss research issues. Then we review related works following with our approaches being presented. Finally we analyze results and conclude the work.

\section{ISSUES IN MOROCCAN HoSPITALS}

Among problems faced in hospitals, we list them here. The time the nurse responds to the call of patients and the time she can reach the doctor. Sending a message often involves delays and waiting time considerably. In addition, nurses cannot be sure if the doctors receive the message or not. Another problem is that nurses cannot know sometimes if the patient took the medication or not. Furthermore, when a doctor wanted to obtain crucial information with a col- league, he had to find a telephone to contact the operator of the hospital and ask them to call the doctor. With the new wireless technology, doctors have direct lines of communication and instant access to patients, which can speed up decisions regarding care and improve the collaboration between the various health professionals.

\section{RELATED WORKS}

Some researchers thought of implementing "Mobile Intelligent Remote-access Healthcare Management System" [2] that is used to integrate a medical knowledge base and patient data. This intelligent wireless remote healthcare monitoring system unifies the communication in case of emergency and a need of doctors. However, the Wireless Sensor Networks communicate over short distance.

Frequency devices are suggested to be used in future since medical employees will access electronically to medical records from any location [3]. Many of the significant research done in unifying communication in hospitals were advanced; however, not all of them are related to patients. Hundreds of patients die in hospitals because of medical errors. Many errors occur due to the lack of information provided. Although the PDA technology cannot avoid all the errors, but at least it will provide information when nurses are exchanged.

Each nurse will be responsible of a specific number of patients. In the database of the wireless mobile, they will be access to check if patients took their medications or not. Some wireless technologies include the use of location tracking [4] intelligent devices, user interfaces, and body sensors [5]. Most of the wireless systems include telecardiology, tele-radiology, and tele-psychology [6]. By using wireless mobile and databases, nurses will be alerted in case a patient needs to take medication. A requirement model for delivering alert messages is used to facilitate communication between employees [7].

The wireless mobile messages will be transformed automatically without a gap time. A mobile tele-cardiology model involved a significant compression ratio and reduction in transmission time over GSM network [8]. Wireless LANs monitor mobiles and stationary patients inside and outside hospital [9]. WLANs are being used in healthcare systems such as Bluetooth technologies [10].

\section{Approaches}

\section{A. Wireless Technology}

The wireless technology allows health professionals to 
stay in touch in real time, which is to the benefit of patients. Doctors at the hospital already earned time and worked more efficiently by using mobile devices for graphics systems for recording on file and they will soon go wireless with the results of examinations of the patients. The first step towards wireless has been the installation of access points throughout the hospital. Doctors and nurses can now use mobile phones and portable devices in the entire property in a secure way.

The wireless network secure supply to physicians and other health care professionals quick and easy access to important information on patients and allow them to communicate with each other from one end to another in the hospital through the mobile devices, which they save time and improve patient care.

\section{B. Knowledge Management}

Healthcare centres, such as hospitals and doctors' offices, must maintain accurate records to ensure effective patient care. A simple mistake can cost someone's life. As a result, doctors and nurses must carefully record test results, physical data, pharmaceutical orders, and surgical procedures. This paperwork often overwhelms healthcare staff, taking 50-70 percent of their time. The use of a mobile data collection device that wirelessly transmits the data to a centralized database significantly increases accuracy and raises the visibility of the data to those who need the information. This result is in better care given to patients.

The use of electronic patient records, with the ability to input, view, and update patient data from anywhere in the hospital, increases the accuracy and speed of healthcare. This improvement is possible by providing each nurse and doctor with a wireless pen-based computer, such as a tablet or PDA, coupled with a wireless network to databases that store critical medical information about the patients.

Most importantly, though, it ensures that hospital staff should be able to administer the right drug to the right person in a timely fashion. This would not be possible without the use of wireless networks to support a centralized database and mobile data collection devices.

\section{System Architecture}

Our research consists of three parts. The first part is a business part. The research question would be: What are the assets of this application and what will bring to hospitals? We conduct a new business application that is knowledge management in hospitals. Then, we estimate the benefits of hospitals using this business application. The second part is a software part where it consists of the design of interfaces using programming language, ASP.Net, and the database of patients' management using SQL server 2005. The software should be simple and clear for all users. Users should not face any problems understanding the use of the web application. The interfaces have to be clear, simple, concrete, and concise. The other part is a wireless network part using the configuration of the WIFI in order to facilitate the communication between employees to provide the good health for patients. Wwireless network will make the communication easiest throughout the personal digital assis- tants.

\section{ANALYSIS AND RESUltS}

The key factors of any healthcare model to success today are quality of knowledge capital available to institutions both from internal and external resources and how they can control this to efficiently manage their health services and systems.

The main objective of knowledge management is to reduce medical errors, to increase patients' safety, and to link all the departments between each other using portable mobile. Using wireless computers containing electronic medical records, nurses display an online work list on the computer. An alert appears on the computer screen, warning the nurse and preventing a potentially fatal error.

The future of the healthcare industry is indeed based on the ability to implement healthcare IT solutions such as wireless communication for more efficiency, better patient care and cost effectiveness. Wireless communication and medication management are keys for reaching these goals. The implementation and success of the project can be used as an example for the industry.

Requirements engineering is the process of discovering the purpose for which it was intended by identifying the needs of client. Private hospitals had been used many system to meet their business, but they still need an advanced one to better meet the needs of patients. After some researches and interviews, we came up with the functional requirements followed with non functional requirements, and the domain requirements.

\section{A. Functional Requirements}

- Patients Management: The client need a function that manages patients at hospitals since the main focus is patients. This function may add patients, modify patients, search for patients, and delete patients, and view file and history of patients.

- Personal Management: The client needs also a function that manages medical staff. This function adds a new staff, modify file of staff, delete staff, view a staff that manages a service, and view a doctor responsible for a specific patient.

- Specialty Management: Each doctor has a specialty and the client needs a function that manages the specialty of doctors. The function permits to view and to add the specialty of doctors.

- Health Management: The client needs a function that manages the health of each patient. This function list the illness of the patient, the medication that the patient did use and is using actually, and the results of laboratory.

- Service Management: The client needs a function for services where it manages the services by listing all services in hospitals, the doctors responsible for the service and also the manager of each service.

- Periods Management: The client needs a function that can keep in touch with the period each patient did spend in hospital. 
- CCAM Management is the common classification of medical acts management. This function consists of adding comments on what's the illness or injuries of each patient in the hospital, adding comments on the act, modifying comments. It mainly deals with patients' illnesses.

- Message Management: This function send alerts and notification to medical nurses in order to remind to give medications or to send medical results. This function poop ups a short message.

- Security Management: The client wants a function that protects the system from any damages by creating accounts, modifying an existing account, and controlling the access to the software.

\section{B. Non Functional Requirements}

- A Graphical User Interface

- Network Configuration

- SQL Server Management

- Database Designer

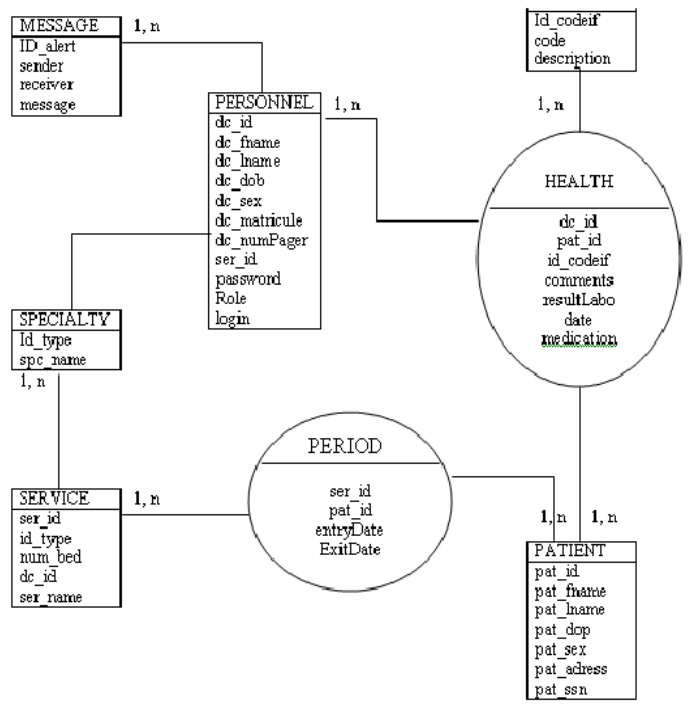

Fig. 1 Conceptual Data Model of Hospitals Management

The context diagram in figure 1 is a general representation of the entire system. The system under investigation is represented as a single process, connected to external entities by data flows and resource flows. The context diagram clearly shows the interfaces between the system under investigation and the external entities with which it communicates. Therefore, it is often conceptually. It serves to focus attention on the system boundary and can help in clarifying the precise scope of the analysis. The processes implemented in our system are: patient management, doctor management, service management, period management, specialty management, health management, and CCAM (common classification of medical acts) management.

- Manage patients is a description of the attributes of the patients. It can perform the operations such as add a patient, delete an existing one, search a patient, view a specific patient, view all patients, and modify a patient. This process could be accessed by both the nurse and the doctors.

- Manage personal used by the manager. It performs the operations add doctor, delete doctor, modify doctor, view doctor's description. It has also Search doctor sub process which is not mentioned in the requirements since it is used only by the local sub processes.

- Manage service is a description of the attributes of services. It can perform the following operations: View the service of each doctor and a nurse, search from a specific service, modify in a service. This process can be accessed by the manager.

- Manage periods is keeping track of the exit and the entry date of patients by freeing patients' beds and displaying the status of patients. This process should be accessed by the manager.

- Manage security allows the restriction of access to the modules by the authentication of each user. For this purpose, each specific user is asked to enter his user name and password. The manager is the only one who has the privilege of accessing all modules and also modifying, creating, deleting accounts and viewing information about users as well. This process uses data from Users' account archive.

- Manage specialty is a process used to set up for each medical staffs a service where they work in with their specialty domain to not complex the research and to automatically execute the research. This process should be managed by the manager.

- Manage health is a process that is designed to allow the different users of the system, nurses, to consult the daily reports to check the patients they need to consult for medicals to avoid errors of distributing the medicines. It also displays alerts concerning the patients that they need medical tests or taking medicines. This process should be managed by both doctors and nurses.

- Manage CCAM is a process that has been designed to produce a list of coded labels, common to the public and private sectors replacing the classification of professional procedures and the catalogue of medical procedures. It has to contain the description of patients' illness and the type of illnesses. This process is managed by both the nurses and doctors.

- Manage message is a process that display alerts when it is sent either to doctors or nurses. This process is used by both the nurses and the doctors. When the alerts are sent it appears on the screen, then you can check it by viewing alerts.

In order to better make the application successful to the needs of the customers, patients. The steps of task are analyzed. The first question was who is going to use the system? The system can be very useful and helpful to nurses for the people who have to take a lot of medication per day. It is convenient to write down reminders and send alerts to nurses in order to avoid errors and medical mistakes. Also, disabled or old people that they never follow what nurses are doing during their treatments would benefit from the wireless application. In general, the device can be useful for 
anybody with allergies, anybody taking pills, and anybody who is not visually impaired.

When considering the potential customers, we have to keep in mind their background skills. The PDA is targeted towards a broad range of medical staff. They do not have to own any device, since our interface comes in its own PDA. They need to have some basic understanding of how to operate everyday devices. The most important thing will be to make the device as simple as possible, so as not to require any background skills from the medical staffs.

Some of the tasks that nurses have to perform right now are listed: One of them would be an automatic reminder that will tell which medicine should be given to patients, and when it is time to restock it. Also, with the PDA, it is very easy to access an inventory of current medication that one takes, which is more convenient than going through the paper work and look for the file of patient to see if the patient took the medications or not, and this may result to errors. Another easy task would be retrieving patient's medical history, which will be stored on the PDA and will help determine if a current medication is compatible with patient's allergies. The PDA interface should be selfexplanatory. The interface will be easy to follow. Just by looking on the screen, they can click the button, which will take them to the next step of the task. Also, each screen will contain a help button in case the customer needs more explanation of a certain screen.

\section{Alerts Management}

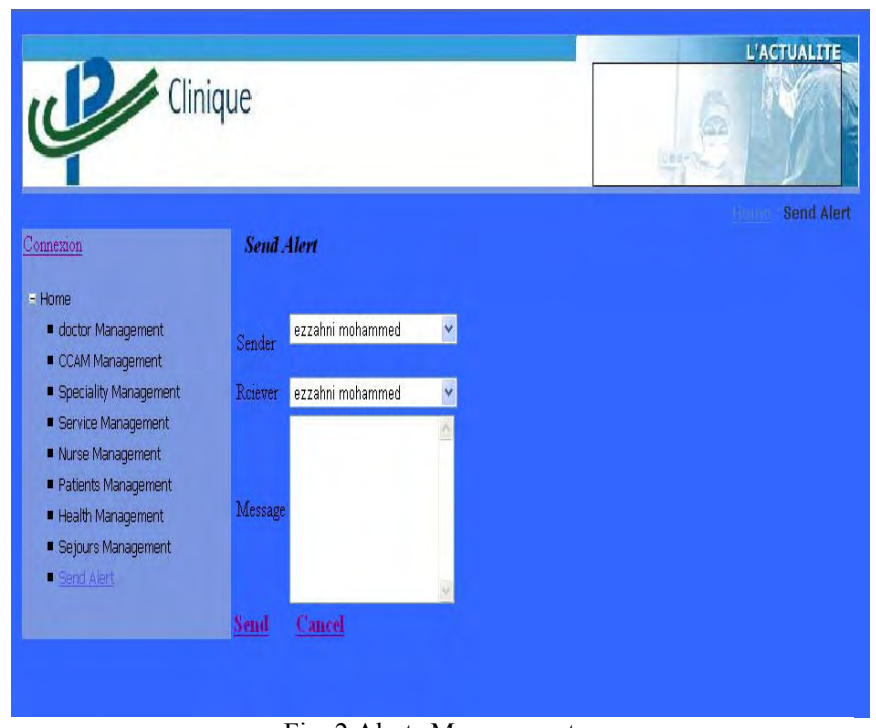

Fig. 2 Alerts Management

Alerts Management is the main process that distinguishes this research from others. When the requests are sent to a specific employee, it appears as an alert in the screen. A medical request is generated as a default alert; when the patient needs a medication in a specific time, this default alert appears on screen as a vibration to aware the nurse to give the medication to avoid errors. Once the patient took the medication, the nurse must record that in database.

WIFI is the contraction of two words wireless fidelity. It is now wide spread. When the term WIFI is published, some manufacturers and retailers have associated with equipment that will not bear the technology IEEE 802.11, but it is compatible with a technique for wireless network. In practice, to use a computer network WIFI, it is necessary to have at least two WIFI equipments such as a computer and a WIFI router. The computer must be equipped with a WIFI which contains an antenna, and allow pilots to run this card.

The standard 802.11 seeks to define the lower layers for a wireless connection using electromagnetic waves. The physical layer (PHY layer sometimes recorded), offering three types of information coding and data link layer, which consists of two sub-layers that is composed of: The control of the logical link (Logical Link Control, or LLC); the medium access control (Media Access Control, or MAC).

The physical layer defines the modulation of radio waves and characteristics of signs for data transmission, while the data link layer defines the interface between the bus of the machine and the physical layer, including an access method similar to that used in standard Ethernet and the rules of communication between the different stations. Within the configuration of the wireless network, we are using the norm IEEE 802.11 that is actually the standard initial offering speeds of 1 or $2 \mathrm{Mbps}$. The objectives are to extend a network cable by Secure WiFi and to enable authorized computer equipped with a wireless network card to connect to an existing network a sharing connection.

The materials used are: Modem USB / Ethernet ADSL, $\mathrm{PC}$ acting as Internet gateway, access point Wifi EUSSO GL2454-API, mobile device with PCMCIA EUSSO GL2454-01, and a switch or hub (10/100Mb/s)

\section{CONCLUSION}

Medical health knowledge management system using wireless network is an innovative project for hospitals to benefit from the wireless networking to gain and please customers through better service. Hospitals and medical health services are large business that we have to make them successful for their earned profits and incomes. Unifying the communication in hospitals can approve the environments and avoid the problems faced.

\section{REFERENCES}

[1] Ng, H S, M L Sim, and C M Tan, (2006) Security issues of wireless sensor networks in healthcare applications, BT Technology Journal 24 (2), pp 138-144.

[2] Qureshi, B., and Tounsi, Mohammed (2006) A Wireless Sensor based Intelligent Mobile System for Remote Healthcare Monitoring in Saudi Arabia, Saudi Arabia.

[3] Nelson L, (1999) Step-by-step guide to selecting mobile wireless devices, Nursing Management 30, (11), pp $12-$ 13.

[4] Koshima H, Hoshen J (2000) Personal locator services emerge. IEEE Spectrum, pp 41-48, Feb 
[5] Bhargava A, Zoltowski M (2003) Sensors and wireless communication for medical care. In Proc. 14th International Workshop on Database and Expert Systems Applications, pp 956-960

[6] Boric-Lubecke O, Lubecke VM (2002) Wireless house calls: Using communications technology for health care and monitoring. IEEE Microw Mag 43-48, Sept

[7] Kafeza E, Chiu DKW, Cheung SC, Kafeza M Alerts in mobile healthcare applications: requirements and pilot study. IEEE Transactions on IT in Biomedicine 8(2):173181
[8] Istepanian R, Petrosian A (2000) Optimal zonal wavelet-based ECG data compression for a mobile telecardiology system. IEEE Trans on IT in Biomedicine 4(3):200211, Sept

[9] Varshney U, Malloy A (2001) On improving the dependability of wireless networks, in Proc. IEEE International Global Communications Conference, Nov (full paper in CD-ROM)

[10] Yu S, Cheng J (2005) A wireless physiological signal monitoring system with integrated bluetooth and $\mathrm{WiFi}$ technologies. In Proc. of IEEE 27th Annual International Conference of the EMBS, pp 2203-2206, Sept 